\title{
Lymph node upstaging for non-small cell lung cancer after uniportal video-assisted thoracoscopy
}

\author{
Mahmoud Ismail ${ }^{1}$, Dania Nachira ${ }^{2}$, Marc Swierzy ${ }^{1}$, Gian Maria Ferretti ${ }^{2}$, Julianna Paulina Englisch ${ }^{1}$, \\ Ramin Raul Ossami Saidy ${ }^{1}$, Feng Li $^{1}$, Harun Badakhshi ${ }^{3}$, Jens C. Rueckert ${ }^{1}$ \\ ${ }^{1}$ Competence Center of Thoracic Surgery, Department of Surgery, Charité - Universitätsmedizin Berlin, Berlin, Germany; ${ }^{2}$ Department of General \\ Thoracic Surgery, Fondazione Policlinico Universitario “A. Gemelli”, Rome, Italy; ${ }^{3}$ Department of Clinical Radiation Oncology, Ernst von \\ Bergmann Medical Center, Potsdam, Germany \\ Contributions: (I) Conception and design: M Ismail; (II) Administrative support: H Badakhshi, JC Rueckert; (III) Provision of study materials or \\ patients: M Swierzy, JC Rückert; (IV) Collection and assembly of data: F Li, GM Ferretti, M Swierzy, JP Englisch, RR Ossami Saidy; (V) Data \\ analysis and interpretation: D Nachira, M Ismail; (VI) Manuscript writing: All authors; (VII) Final approval of manuscript: All authors. \\ Correspondence to: Mahmoud Ismail, MD. Competence Center of Thoracic Surgery, Department of Surgery, Charité-Universitätsmedizin Berlin, \\ Charitéplatz 1, Berlin 10117, Germany. Email: mahmismail@gmail.com.
}

Background: Radical lymph node dissection (LND) plays a major role in the treatment of non-small cell lung cancer (NSCLC). This study presents the analysis of the results after uniportal video-assisted thoracoscopy (VATS) lymphadenectomy during anatomical lung resections for NSCLC, focusing on pathological nodal upstaging. Any possible risk factor affecting nodal upstaging was also investigated.

Methods: The prospectively collected clinical data of 136 patients undergone uniportal VATS anatomical lung resections, from June 2012 to September 2017, were reviewed. In particular, all details inherent the clinical and pathological node stage and any possible risk factor affecting nodal upstaging were analyzed.

Results: The patient population consisted of 90 males and 46 females; their mean age was $67.42 \pm 10.64$ years. The mean number of lymph nodes retrieved during uniportal VATS lymphadenectomy was $20.14 \pm 10.73$ (7.27 \pm 5.90 and $12.60 \pm 7.96$ in N1 and N2 stations, respectively). The incidence of nodal upstaging was $13.3 \%$ (18 cases). In particular there was a N0-1 upstaging in 10 cases (7.4\%), a N1-2 upstaging in 3 (2.2\%) and a N0-2 in 4 (3\%). The ROC analysis showed that the resection of 18 lymph nodes was the best predictor of a general upstaging with an AUC-ROC of 0.595, while the resection of 7 hilar lymph nodes was the best predictor of N1 upstaging (AUC-ROC: 0.554) and 11 mediastinal nodes was the best predictor of N2 upstaging (AUC-ROC: 0.671). The number of positive lymph nodes of stations 5-6 (OR: 2.035, 95\% CI: 1.082-3.826, $\mathrm{P}=0.027$ ) and stations 2-3-4 (OR: 6.198, 95\% CI: 1.580-24.321, $\mathrm{P}=0.009$ ) were confirmed to be the only independent risk factors for $\mathrm{N} 2$ upstaging by multivariate analysis.

Conclusions: According to our experience, uniportal VATS allows a safe and effective radical lymphadenectomy, with a satisfactory pathological nodal upstaging, comparable to other minimally invasive techniques.

Keywords: Uniportal video-assisted thoracoscopy (VATS); lymph node upstaging; lung cancer; lung major resections

Submitted May 07, 2018. Accepted for publication Jun 11, 2018.

doi: $10.21037 /$ jtd.2018.06.70

View this article at: http://dx.doi.org/10.21037/jtd.2018.06.70 


\section{Introduction}

In the last 20 years, minimally invasive surgical techniques have been increasingly used for lung resections for nonsmall cell lung cancer (NSCLC) and have shown superior post-operative outcomes when compared to thoracotomy: shorter hospitalization, less post-operative pain, preserved pulmonary function and faster access to possible adjuvant therapies (1). To validate these techniques, several studies have focused on the radicality of lymph node dissection (LND) and on the prevalence of nodal upstaging to evaluate the relative efficacy and oncologic equivalence of open versus minimally invasive surgery.

Nodal upstaging in patients with NSCLC is defined as the unexpected pathological finding of metastasis in hilar (pN1) or mediastinal (pN2) lymph nodes in patients who were presumed to have clinical $\mathrm{cN} 0$ or $\mathrm{cN} 1$ at pre-operative staging exams (2). Therefore, the incidence of nodal upstaging is considered a quality criterion of completeness of lymphadenectomy.

Several studies in the literature have treated the upstaging topic in different techniques comparing VATS, Robotic Assisted Thoracic Surgery (RATS) and thoracotomy with discordant results. Some studies as the one by Sugi et al. (3), Watanabe et al. (4), Stephens et al. (5) and D'Amico et al. (6) showed no evidence of differences in survival and in number of lymph nodes removed between VATS and thoracotomy. On the contrary, other studies as the one by Boffa et al. (7) and Medbery et al. (8) concluded that upstaging was more frequent in the thoracotomy group. Regarding RATS Cerfolio et al. (9) demonstrated that lymphadenectomy was similar to thoracotomy. On the other hand, Wilson et al. (10) reported that the rate of nodal upstaging was higher in RATS versus VATS but with similar disease-free and overall survival. Only a recent study by Martin et al. (11) compared the prevalence of upstaging in VATS, RATS and open surgery and concluded that the prevalence of nodal upstaging was less in VATS and RATS than in thoracotomy.

At the moment in the literature there are no studies that have investigated the incidence of lymph node upstaging in patients who have undergone uniportal VATS anatomical lung resection.

The aim of this study is to evaluate the efficacy of LND performed with the uniportal VATS technique, through the determination of pathological nodal upstaging in our series. Any possible risk factor affecting nodal upstaging was also investigated.

\section{Methods}

The prospectively collected clinical data of 642 patients undergone uniportal VATS resections, from June 2012 to September 2017 at the Thoracic Surgery Department, Charité - Universitätsmedizin Berlin (Germany), were retrospectively reviewed.

Among these patients, 136 patients undergone anatomical lung resections were selected, after excluding patients operated on for diseases other than NSCLC or undergone wedge resections. Patients undergone preoperative neoadjuvant treatment were also excluded for avoiding any confounder factor.

The records of all 136 patients were reviewed, with particular attention to details regarding the clinical and pathological stage, the total number and the number of positive lymph nodes retrieved per each station and factors possibly affecting the pathological downstaging and upstaging [histology, Standard uptake value (SUV) at PETCT, type of anatomical resection, tumor localized in upper or lower lobe, side, size of the tumor bigger than $2 \mathrm{~cm}$, comorbidities, etc.].

All patients provided written informed consent before operation for the treatment of their clinical data and underwent preoperative evaluation, including: routine blood tests, electrocardiography, radiological and diagnostic examinations [total body computed tomography (CT), PET-CT, Endobronchial ultrasound with biopsies...] and pulmonary function test.

\section{Preoperative staging system}

Routine invasive staging with EBUS biopsies was carried out in each case where CT-scan or PET showed evidence of $\mathrm{N} 2$ enlargement or involvement in order to clarify the real extension of disease.

\section{Surgical technique}

The same team performed all procedures under general anesthesia and single-lung ventilation. The operations were performed according to our standardized steps (12). The same principles for oncological radical LND were preserved as in standardised open surgery. The radical LND included the lobe related N1 hilar positions 10 and 11 . The radical LND for N2 was performed for the positions 7, 8, 9 as well as 2, 3, 4 for right side or 5, 6 for left side. For the radical LND special uniportal VATS instruments and energy 
Table 1 Clinical characteristics of patients and types of tumor and lung resection

\begin{tabular}{lc}
\hline Characteristics & Value $(\mathrm{n}=136)$ \\
\hline Smoker & $120(88.2 \%)$ \\
ASA score & $2.56 \pm 0.62$ \\
Cardiovascular diseases & $42(30.9 \%)$ \\
Respiratory diseases & $41(30.1 \%)$ \\
Kidney diseases & $18(13.2 \%)$ \\
DM II & $31(22.8 \%)$ \\
Preop FEV1\% & $79.27 \pm 19.36$ \\
Side (right) & $81(59.6 \%)$ \\
Tumor size (mm) & $27.05 \pm 17.35$ \\
Operative time (min) & $248.97 \pm 118.17$ \\
Histology & \\
Adenocarcinoma & $91(66.9 \%)$ \\
Squamous cell carcinoma & $39(28.7 \%)$ \\
Carcinoid tumors & $6(4.4 \%)$ \\
Type of resection & $18(13.2 \%)$ \\
Segmentectomy & $2(1.5 \%)$ \\
Lobectomy & \\
Bilobectomy & \\
Pneumonectomy & \\
\hline & \\
\hline
\end{tabular}

device were used.

\section{Statistical analysis}

Categorical variables are reported as $\mathrm{n}(\%)$. Continuous variables are expressed as mean \pm standard deviation (SD). Categorical variables were compared by Fischer's exact test and continuous variables by independent sample Student's $t$-test. ROC Curve analysis was performed to assess the cut-off points that provided the best predictors (number of nodes) for downstaging and upstaging per each lymph nodal station. Univariate logistic regression was performed to evaluate any possible risk factor for nodal upstaging. Any variable with a $\mathrm{p}$ value less than 0.20 at univariate analysis was selected for inclusion in a multivariate analysis. Forward stepwise logistic regression analysis was used to select the independent risk factors for lymph nodal upstaging.

A $P$ value less than 0.05 was considered statistically
Table 2 Total number and number of positive lymph nodes (+) per each station

\begin{tabular}{lcc}
\hline Station & Total number & Positive number \\
\hline $2-4$ & $5.19 \pm 6.23$ & $0.06 \pm 0.37$ \\
$5-6$ & $1.99 \pm 3.45$ & $0.13 \pm 0.82$ \\
7 & $3.86 \pm 3.66$ & $0.12 \pm 0.61$ \\
$8-9$ & $1.70 \pm 2.05$ & $0.03 \pm 0.24$ \\
10 & $6.91 \pm 5.77$ & $0.42 \pm 1.43$ \\
11 & $0.41 \pm 1.42$ & $0.01 \pm 0.09$ \\
\hline
\end{tabular}

significant. Statistical analysis was performed using IBM SPSS Statistics for Macintosh, Version 25.00 (Armonk, NY, USA).

\section{Results}

The patient population consisted of 90 males and 46 females; their mean age was $67.42 \pm 10.64$ years. The majority of study population was operated for an Adenocarcinoma (91 cases, 66.9\%) and the main type of uniportal VATS anatomical lung resection was lobectomy (114 cases, $83.8 \%$ ) followed by segmentectomy (18 cases, $13.2 \%)$. The clinical stage was: stage I in 104 patients (76.5\%), stage II in 15 (11\%), stage III in $15(11 \%)$ and stage 4 (oligo-metastatic cancer) in 2 patients $(1.5 \%)$. Clinical characteristics of patients, comorbidities and types of tumor and lung resection are shown in Table 1.

The mean number of lymph nodes retrieved during uniportal VATS LND was $20.14 \pm 10.73(7.27 \pm 5.90$ in N1 stations and $12.60 \pm 7.96$ in N2). In general, a N1 nodal metastatization was pathologically found in about $0.42 \pm 1.25$ nodes, while in $0.29 \pm 1.15$ nodes in $\mathrm{N} 2$ stations (the distribution of nodal positivity per station is reported in Table 2).

Among the $13.3 \%$ of postoperative complications (18 cases, mainly prolonged air-leak, pneumonia, postoperative atelectasis, etc.), it was recorded only one case of chylothorax and no case of recurrent nerve injury.

In our series, a pathological downstaging was recorded in 16 cases $(11.9 \%)$; all possible risk factors affecting downstaging were evaluated and histology other than Adenocarcinoma resulted the only one involved in a statistical positive way $(\mathrm{P}=0.038)$.

Furthermore, according to the ROC analysis, the resection of 14 lymph nodes was the best predictor of 


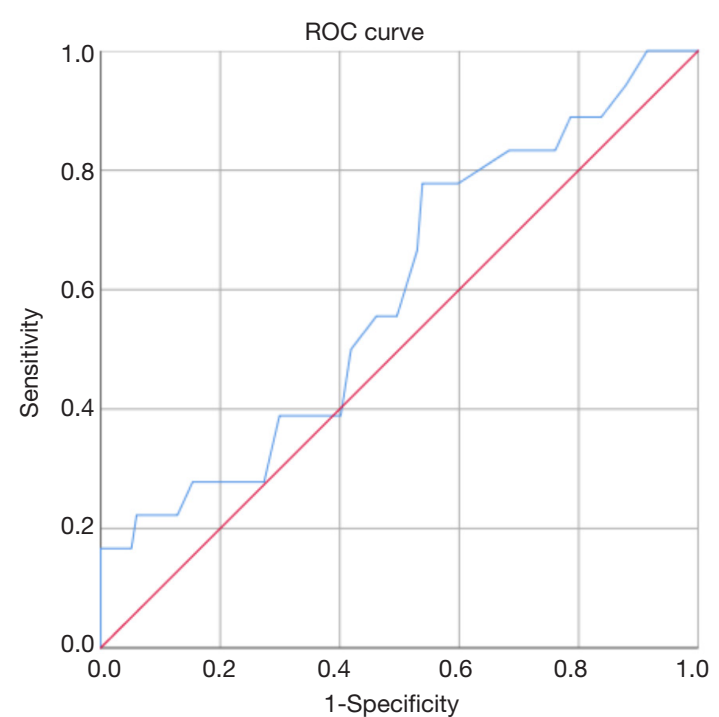

Figure 1 ROC Curve of the impact of total number of lymph nodes removed on upstaging (AUC-ROC: 0.595).

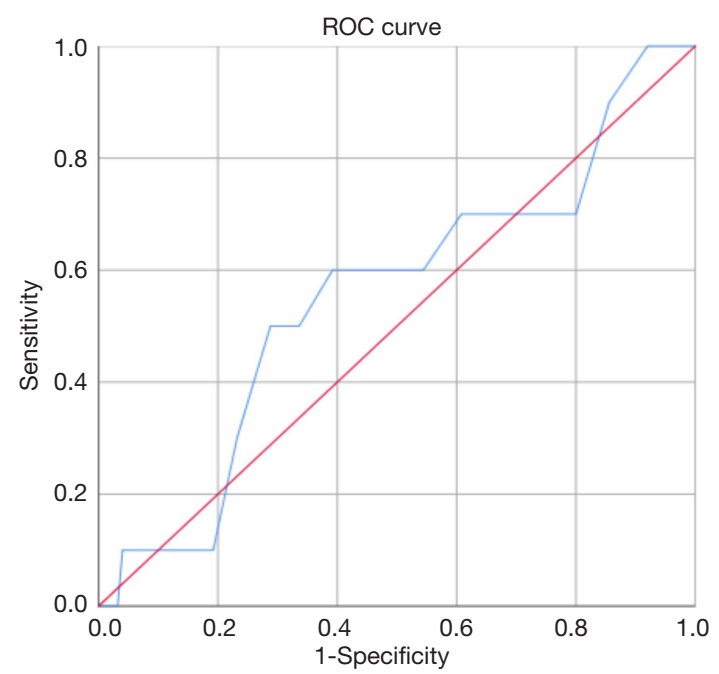

Figure 2 ROC Curve of the impact of number of hilar lymph nodes removed on N1 upstaging (AUC-ROC: 0.554).

downstaging [area under curve (AUC): 0.38].

On the contrary, the incidence of nodal upstaging was $13,3 \%$ (18 cases). In particular there was a N0-1 upstaging in 10 cases $(7.4 \%)$, a N1-2 upstaging in $3(2.2 \%)$ and a N0-2 in $4(3 \%)$.

The ROC analysis showed that, in general, the resection of 18 lymph nodes was the best predictor of upstaging with an AUC-ROC of 0.595 (Figure 1), while the resection of 7

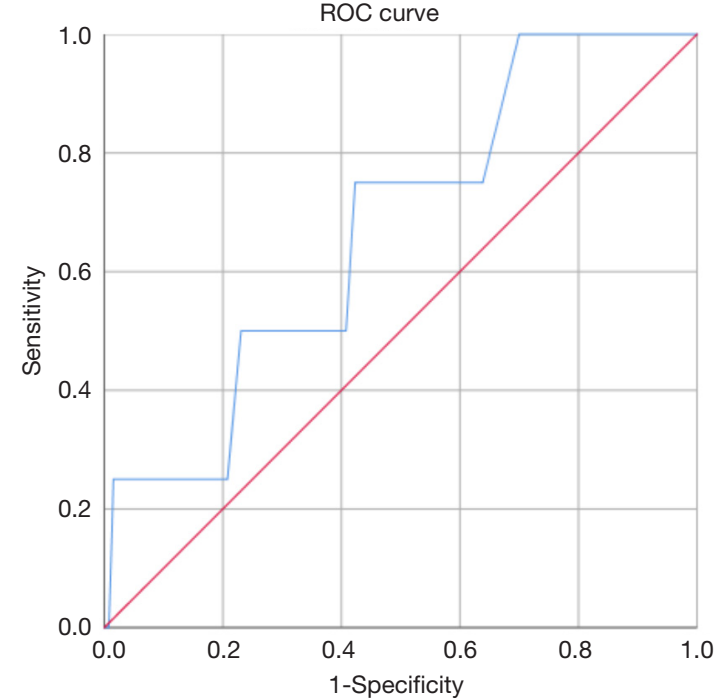

Figure 3 ROC Curve of the impact of number of mediastinal lymph nodes removed on N2 upstaging (AUC-ROC: 0.671).

hilar lymph nodes was the best predictor of $\mathrm{N} 1$ upstaging (AUC-ROC: 0.554, Figure 2) and 11 mediastinal nodes was the best predictor of N2 upstaging (AUC-ROC: 0.671, Figure 3).

Evaluating any possible risk factor for nodal upstaging in general, it was found only a trend toward statistical significance $(\mathrm{P}=0.084)$ in case of patients affected by Diabetes Mellitus II.

On logistic regression, the N1 upstaging was not correlated with the number of nodes removed or the number of positive nodes per each hilar station (10 and 11).

Meanwhile, the N2 upstaging was correlated with the number of positive lymph nodes in stations $2-4(\mathrm{P}=0.006)$, 5-6 $(\mathrm{P}<0.001), 7(\mathrm{P}=0.047)$ and $8-9(\mathrm{P}=0.004)$. The multivariate analysis confirmed the number of positive lymph nodes of stations 5-6 (OR: 2.035, 95\% CI: $1.082-$ 3.826, $\mathrm{P}=0.027)$ and stations 2-4 (OR: 6.198, 95\% CI: $1.580-24.321, \mathrm{P}=0.009)$ as the only independent risk factors for N2 upstaging.

\section{Discussion}

Stage migration for NSCLC is an underestimated issue. Lymph node upstaging is defined as the presence of unsuspected pathologic hilar (pN1) or mediastinal (N2) lymph nodes in the final histopathological specimen $(2,11)$. This is very important to evaluate because if upstaging happens, the patient does need adjuvant chemotherapy in 
most of the cases. Then there is a direct correlation to the patient's survival. Lymph node upstaging is to be considered as an instrument of quality of lung cancer surgery.

The preoperative lymph node staging for NSCLC is standardized and described in several papers and guidelines $(6,13,14)$. It is performed through radiological instruments (CT, PET-CT) or invasively (EBUS, mediastinoscopy) (15). The failure of preoperative staging in NSCLC is well investigated. Referring to stage I NSCLC, around $28 \%$ of the preoperative staging is incorrect $(13,15)$. The invasive staging is more correct than non-invasive but shows also more complications $(13,15)$. Especially the hilar and peribronchial staging is a technical challenge.

Nodal upstaging is related to the number of nodes removed (16-18). In different countries, a different number of lymph nodes for pathological evaluation is recommended. Zhong et al. (17) mentioned that a lymphadenectomy of at least ten nodes should be performed.

The European Society of Thoracic surgery (ESTS) guidelines recommend to perform the lymphadenectomy as intense as possible, also because of the potential presence of skip metastases. If ever possible a systematic nodal dissection is recommended: $2 \mathrm{R}$ and $4 \mathrm{R}$ and en-bloc resection of the lower mediastinal nodes (stations 7, 8, and 9) should be performed for right side, while on the left side removal of the subaortic (station 5), para-aortic (station 6), station 7, 8 and 9 are required to be removed (14).

Till now, the level of evidence of VATS superiority in lymphadenectomy has been low and there is not always concordance in the results proposed by studies of different groups.

Sugi et al. (3), Watanabe et al. (4), Stephens et al. (5), D'Amico et al. (6) showed no evidence of differences in survival and in number of lymph nodes removed between VATS and thoracotomy.

On the contrary, other studies as the one by Boffa et al. (7), conducted on 11,513 patients, concluded that upstaging from $\mathrm{N} 0$ to $\mathrm{N} 1$ is more common in thoracotomy versus VATS surgery (9.3\% and $6.7 \%$, respectively). However, upstaging from N0 to N2 was similar (5\% and $4.9 \%$, respectively). Medbery et al. (8) analyzed 16,983 patients from the National Cancer database and concluded that despite the quantity of lymph nodes removed in VATS was higher than in open surgery (10.42 versus 9.44), upstaging was more frequent in the thoracotomy group (12.8\% vs. $10.3 \%)$.

Regarding the Robotic Assisted Thoracic surgery (RATS), Cerfolio et al. (9) demonstrated that there weren't any differences in the number of lymph nodes removed between RATS and Thoracotomy. Instead, Wilson et al. (10) reported that the rate of nodal upstaging was higher in RATS $v s$. VATS but with similar disease-free and overall survival.

Martin and colleagues (11) analyzed 2,830 patients subjected to lung resection, included in the Kentucky Cancer Registry database from 2010 to 2012, and documented an overall prevalence of nodal upstaging of $8.8 \%$. In particular nodal upstaging in the thoracoscopy group was $4.8 \%$, in RATS group was $8.6 \%$ and in open surgery group was $9.9 \%$. The Authors emphasized, however, that thoracoscopy was associated with lower morbidity and an improved survival in pathologic stage I.

Interestingly, a recent study from Toker et al. (19) compared the effectiveness of LND in VATS, RATS and open surgery together: the three techniques had similar results in number of lymph nodes removed but RATS showed to dissect more nodes in N1 stations.

To the best of our knowledge, this is the first paper that widely analyzes the results of lymphadenectomy and in particular the nodal upstaging exclusively in uniportal VATS technique.

The results in our series showed that the mean number of lymph nodes retrieved during uniportal VATS was $20.14 \pm 10.73$, a satisfactory value compared to the mean reported in literature $[13.42 \pm 8.24$ VATS (20), 9.44 in open surgery (8), 17 in RATS (9)].

Furthermore, the incidence of nodal upstaging (13.3\%), was comparable or even superior to that of other techniques $(6.7-12.8 \%)(8,9,11,20)$.

In particular the N0-1 (7.4\%), N1-2 (2.2\%) and N0-2 (3\%) upstaging were also in line with the literature (7-9).

Recently, several studies $(7,21-24)$ pointed some risk factors out for nodal upstaging: some of them could be related to patient history (diabetes mellitus, rheumatoid arthritis or other pathologies) or to tumor characteristics (localization of tumor, T stage, SUVmax value or histology).

From the analysis of any possible correlation between pathological node staging and risk factors that was carried out in our series, it was found that the main risk factor for nodal downstaging was histology other than Adenocarcinoma $(\mathrm{P}=0.038)$, while for nodal upstaging there was only a trend toward statistical significance $(\mathrm{P}=0.084)$ in case of patients affected by Diabetes Mellitus II. Moreover, the N2 upstaging was correlated with the number of positive lymph nodes in stations $2-4,5-6,7$ and $8-9$. The multivariate analysis confirmed only the number 
of positive lymph nodes of stations 5-6 and 2-4 as the only independent risk factors for $\mathrm{N} 2$ upstaging.

To summarize, according to our experience, uniportal VATS is a safe and feasible approach also for performing an extended lymphadenectomy. In the hands of expert uniportal surgeons, the rate of nodal upstaging is comparable to that of other minimally invasive approaches. Uniportal VATS can be considered to be an effective and advisable technique for LND.

\section{Acknowledgements}

None

\section{Footnote}

Conflicts of Interest: The authors have no conflicts of interest to declare.

Ethical Statement: This study was evaluated by the Institutional Review Board (IRB) of Charité Universitätsmedizin Berlin and, as this was a retrospective review for service evaluation and there was no modification in patients' care (no prospective randomized study), we did not need the final ethical approval of our IRB. All patients signed a written informed consent before operation for the treatment of their clinical data.

\section{References}

1. Nicastri DG, Wisnivesky JP, Litle VR, et al.Thoracoscopic lobectomy: report on safety, discharge independence, pain, and chemotherapy tolerance. J Thorac Cardiovasc Surg 2008;135:642-7.

2. Toker A, Özyurtkan MO, Kaba E. Nodal upstaging: effects of instrumentation and three-dimensional view in clinical stage I lung cancer. J Vis Surg 2017;3:76.

3. Sugi K., Kaneda Y, Esato K. et al. Video-assisted Thoracoscopic Lobectomy Achieves a satisfactory longterm prognosis in patient with clinical Stage IA Lung Cancer. World J Surg 2000;24:27.

4. Watanabe A, Koyanagi T, Ohsawa H, et al. Systematic node dissection by VATS is not inferior to that through an open thoracotomy: a comparative clinicopathologic retrospective study. Surgery 2005;138:510-7.

5. Stephens N, Rice D, Correa A, et al. Thoracoscopic lobectomy is associated with improved short-termand equivalent oncological outcomes compared with open lobectomy for clinical Stage I non-small-cell lung cancer: a propensity-matched analysis of 963 cases. Eur J Cardiothorac Surg 2014;46:607-13.

6. D'Amico TA, Niland J, Mamet R, et al. Efficacy of mediastinal lymph node dissection during lobectomy for lung cancer by thoracoscopy and thoracotomy. Ann Thorac Surg 2011;92:226-31; discussion 231-2.

7. Boffa DJ, Kosinski AS, Paul S, et al. Lymph node evaluation by open or video-assisted approaches in 11,500 anatomic lung cancer resections. Ann Thorac Surg 2012;94:347-53; discussion 353.

8. Medbery RL, Gillespie TW, Liu Y, et al. Nodal Upstaging Is More Common with Thoracotomy than with VATS During Lobectomy for Early-Stage Lung Cancer: An Analysis from the National Cancer Data Base. J Thorac Oncol 2016;11:222-33.

9. Cerfolio RJ, Bryant AS, Skylizard L, et al. Initial consecutive experience of completely portal robotic pulmonary resection with 4 arms. J Thorac Cardiovasc Surg 2011;142:740-6.

10. Wilson JL, Louie BE, Cerfolio RJ, et al. The prevalence of nodal upstaging during robotic lung resection in early stage non-small cell lung cancer. Ann Thorac Surg 2014;97:1901-6; discussion 1906-7.

11. Martin JT, Durbin EB, Chen L, et al. Nodal Upstaging During Lung Cancer Resection Is Associated With Surgical Approach. Ann Thorac Surg 2016;101:238-44; discussion 44-5.

12. Ismail M, Swierzy M, Nachira D, et al. Uniportal videoassisted thoracic surgery for major lung resections: pitfalls, tips and tricks. J Thorac Dis 2017;9:885-97.

13. Krantz SB, Lutfi W, Kuchta K, et al. Improved Lymph Node Staging in Early-Stage Lung Cancer in the National Cancer Database. Ann Thorac Surg 2017;104:1805-14.

14. Lardinois D, Leyn $P$, van Schil $P$, et al. ESTS guidelines for intraoperative lymph node staging in non-small cell lung cancer. Eur J Cardiothorac Surg 2006;30:787-92.

15. Lewis J, Gillaspie EA, Osmundson EC, et al. Before or After: Evolving Neoadjuvant Approaches to Locally Advanced Non-Small Cell Lung Cancer. Front Oncol 2018;8:5.

16. Toosi K, Velez-Cubian FO, Glover J, et al. Upstaging and survival after robotic-assisted thoracoscopic lobectomy for non-small cell lung cancer. Surgery 2016;160:1211-8.

17. Zhong W, Yang X, Bai J, et al. Complete mediastinal lymphadenectomy: the core component of the multidisciplinary therapy in resectable non-small cell lung cancer. Eur J Cardiothorac Surg 2008;34:187-95. 
18. Gonzalez-Rivas D, Fieira E, Delgado M, et al. Is uniportal thoracoscopic surgery a feasible approach for advanced stages of non-small cell lung cancer? J Thorac Dis 2014;6:641-8.

19. Toker A, Özyurtkan MO, Demirhan Ö, et al. Lymph Node Dissection in Surgery for Lung Cancer: Comparison of Open vs. Video-Assisted vs. Robotic-Assisted Approaches. Ann Thorac Cardiovasc Surg 2016;22:284-90.

20. Gonfiotti A, Bertani A, Nosotti M, et al. Italian VATS Group. Safety of lymphadenectomy during video-assisted thoracic surgery lobectomy: analysis from a national database. Eur J Cardiothorac Surg 2018. [Epub ahead of print].

21. Rocha AT, McCormack M, Montana G, et al. Association between lower lobe location and upstaging for early-stage non-small cell lung cancer. Chest 2004;125:1424-30.

22. Al-Sarraf N, Aziz R, Doddakula K, et al. Factors causing inaccurate staging of mediastinal nodal involvement in non-small cell lung cancer patients staged by positron emission tomography. Interact Cardiovasc Thorac Surg 2007;6:350-3.

23. Lee PC, Port JL, Korst RJ, et al. Risk factors for occult mediastinal metastases in clinical stage I non-small cell lung cancer. Ann Thorac Surg 2007;84:177-81.

24. Decaluwé H, Stanzi A, Dooms C, et al. Central tumor location should be considered when comparing N1 upstaging between thoracoscopic and open surgery for clinical stage I non-small-cell lung cancer. Eur J Cardiothorac Surg 2016;50:110-7.
Cite this article as: Ismail $M$, Nachira D, Swierzy M, Ferretti GM, Englisch JP, Ossami Saidy RR, Li F, Badakhshi H, Rueckert JC. Lymph node upstaging for non-small cell lung cancer after uniportal video-assisted thoracoscopy. J Thorac Dis 2018;10(Suppl 31):S3648-S3654. doi: 10.21037/jtd.2018.06.70 